\title{
RICCATI TYPE OPERATOR EQUATION AND FURUTA'S QUESTION
}

\section{JIANGTAO YUAN AND CAIHONG WANG}

Abstract. As a continuation of the Riccati type operator equation $K=T H T$, this article is to consider the Riccati type equation

$$
K^{p}=H^{\frac{\delta}{2}} T^{\frac{1}{2}}\left(T^{\frac{1}{2}} H^{\delta+r} T^{\frac{1}{2}}\right)^{\frac{1}{w}-1} T^{\frac{1}{2}} H^{\frac{\delta}{2}}
$$

and its applications to Furuta's question. The Riccati type equation is discussed via Douglas's result. As applications, a result by Fujii et al. is extended which implies that the converse of Furuta's question is right, and a result by Lin-Cho is generalized.

Mathematics subject classification (2010): 47A62, 47A63, 47B15, 47B65.

Keywords and phrases: Positive semidefinite operator, Riccati equation, Loewner-Heinz inequality, Furuta inequality.

\section{Introduction}

Throughout this paper, an operator $T$ means a bounded linear operator on a Hilbert space. $T \geqslant 0$ and $T>0$ mean a positive semidefinite operator and a positive definite operator respectively.

The well-known Riccati type operator equations $K=T H T-T C-C^{*} T$ relate to control theory closely and have been studied extensively [14].

Pedersen and Takesaki [16] developed the special kind of Riccati equation $K=$ THT as a useful tool for the noncommutative Radon-Nikodym theorem [17].

Bach and Furuta [1, 7] gave discussions on the equation $K=T\left(H^{\frac{1}{n}} T\right)^{n}$ where $n$ is a natural number.

Yuan and Gao [18] discussed the Riccati type equation

$$
K^{p}=H^{\frac{\delta}{2}} T^{\frac{1}{2}}\left(T^{\frac{1}{2}} H^{\delta+r} T^{\frac{1}{2}}\right)^{\frac{p-\delta}{\delta+r}} T^{\frac{1}{2}} H^{\frac{\delta}{2}} .
$$

and proved the result below.

THEOREM 1.1. ([18]) Let $H \geqslant 0, K \geqslant 0$ and assume that $\operatorname{ker} H=\{0\}$.

(1) The following statements are equivalent for each $p>0, r>0$ and $p \geqslant \delta \geqslant 0$ :

(a) $a H^{\delta+r} \geqslant\left(H^{\frac{r}{2}} K^{p} H^{\frac{r}{2}}\right)^{\frac{\delta+r}{p+r}}$ for some $a \geqslant 0$.

(b) There exists a unique operator $T \geqslant 0$ satisfies $\|T\| \leqslant a$ and (1.1).

If in additional $H$ is invertible, (1.1) holds for $p \geqslant \delta>-r$. 
(2) If there exists $T \geqslant 0$ satisfying (1.1) for fixed $p>0, r>0$ and $p \geqslant \delta \geqslant 0$, then, for $p_{1} \geqslant p$ and $r_{1} \geqslant r$, there exists $T_{1} \geqslant 0$ satisfying

$$
K^{p_{1}}=H^{\frac{\delta}{2}} T_{1}^{\frac{1}{2}}\left(T_{1}^{\frac{1}{2}} H^{\delta+r_{1}} T_{1}^{\frac{1}{2}}\right)^{\frac{p_{1}-\delta}{\delta+r_{1}}} T_{1}^{\frac{1}{2}} H^{\frac{\delta}{2}} .
$$

The case $p=1, r=\frac{1}{n}$ and $\delta=0$ of Theorem 1.1 is the main result of [7]. One of the applications of Riccati equation (1.1) is to show that the inclusion relations among class $A(p, r)$ operators are strict [18, Theorem 3.1].

Inspired by recent developments on operator equations including Sylvester equation (see $[3,11])$, in this paper, we will consider the Riccati type equation

$$
K^{p}=H^{\frac{\delta}{2}} T^{\frac{1}{2}}\left(T^{\frac{1}{2}} H^{\delta+r} T^{\frac{1}{2}}\right)^{\frac{1}{w}-1} T^{\frac{1}{2}} H^{\frac{\delta}{2}}
$$

and Furuta's question. Obviously, the special case $w=\frac{\delta+r}{p+r}$ of (1.3) is just (1.1).

Furuta's question ([10, Q2]). Let $A>0, B>0$. Then $\log A \geqslant \log B$ if and only if

$$
A^{r+t} \geqslant\left(A^{\frac{r}{2}}\left(A^{\frac{t}{2}} B^{p} A^{\frac{t}{2}}\right)^{s} A^{\frac{r}{2}}\right)^{\frac{r+t}{(p+t) s+r}}
$$

holds for $p \geqslant 1,-1 \leqslant t<0, r \geqslant-t$ and $s \geqslant 1$ ?

The case $s=1$ of Furuta's question is clear by [5]. Furuta [10] gave two concrete operators $A$ and $B$ such that $\log A \geqslant \log B$ and (1.4) does not holds for $p=2, t=-1$, $r=2$ and $s=2$. This implies that the converse is essential in the question. When $p=1, t=-1$ and $r=2$, Fujii et al [6, Theorem 1] proved the following result by using Kantorovich type inequality.

Theorem 1.2. ([6]) Let $A>0, B>0$. If

$$
A \geqslant\left(A\left(A^{\frac{-1}{2}} B A^{\frac{-1}{2}}\right)^{s} A\right)^{\frac{1}{2}}
$$

holds for sufficiently large $s>1$, then $A \geqslant B$.

Sine $A \geqslant B$ is stronger than $\log A \geqslant \log B$, Theorem 1.2 implies that, when $p=1$, $t=-1$ and $r=2$, the converse of Furuta's question is right.

In this article, section 2 is devoted to discussions on the equation (1.3), the main result is Theorem 2.3 which is an extension of Theorem 1.1 (1).

In section 3, as applications, we give extensions of Theorem 1.2 which imply that the converse of Furuta's question above is right (see Corollary 3.3), and a generalization of a result by Lin-Cho [15, Theorem 2.1].

\section{Riccati type operator equation}

LEMMA 2.1. ([4]) The following assertions are equivalent for $A$ and $B$.

(1) $A A^{*} \leqslant \lambda B B^{*}$ for some $\lambda \geqslant 0$. 
(2) There exists a $C$ with $A=B C$ and $\|C\|^{2} \leqslant \lambda$.

Lemma 2.2. ([8,9]) Let $\alpha \in R$ and $X$ be invertible. Then

$$
\left(X^{*} X\right)^{\alpha}=X^{*}\left(X X^{*}\right)^{\alpha-1} X,
$$

especially in case $\alpha \geqslant 1$ the equality holds without invertibility of $X$.

THEOREM 2.3. Let $H \geqslant 0, K \geqslant 0$ and assume that $\operatorname{ker} H=\{0\}$. The following statements are equivalent for each $p>0, r>0, \delta \geqslant 0,0<w \leqslant 1$ and $a>0$.

(1) $a H^{\delta+r} \geqslant\left(H^{\frac{r}{2}} K^{p} H^{\frac{r}{2}}\right)^{w}$.

(2) There exists a unique operator $T$ satisfying $0 \leqslant T \leqslant a$ and (1.3).

If in additional $H$ is invertible, the condition $\delta \geqslant 0$ can be replaced with $\delta \in \mathscr{R}$ where $\mathscr{R}$ means the set of all real numbers; and if $H$ and $K$ are both invertible, the conditions $\delta \geqslant 0$ and $0<w \leqslant 1$ can be replaced with $\delta \in \mathscr{R}$ and $w \neq 0$.

The case $w=\frac{\delta+r}{p+r}$ of Theorem 2.3 implies (1) of Theorem 1.1.

Proof. (1) $\Rightarrow(2)$. By Lemma 2.1, there exists an operator $S$ so that

$$
\left(H^{\frac{r}{2}} K^{p} H^{\frac{r}{2}}\right)^{\frac{w}{2}}=H^{\frac{\delta+r}{2}} S=S^{*} H^{\frac{\delta+r}{2}} .
$$

Put $T=S S^{*}$, then $\|T\| \leqslant a$ and by Lemma 2.2,

$$
H^{\frac{r}{2}} K^{p} H^{\frac{r}{2}}=\left(H^{\frac{\delta+r}{2}} T H^{\frac{\delta+r}{2}}\right)^{\frac{1}{w}}=H^{\frac{\delta+r}{2}} T^{\frac{1}{2}}\left(T^{\frac{1}{2}} H^{\delta+r} T^{\frac{1}{2}}\right)^{\frac{1}{w}-1} T^{\frac{1}{2}} H^{\frac{\delta+r}{2}} .
$$

So (1.3) holds for $\operatorname{ker} H=\{0\}$.

(2) $\Rightarrow(1)$. For $a$ with $\|T\| \leqslant a$, by Lemma 2.2, (1.3) implies

$$
\begin{aligned}
\left(H^{\frac{r}{2}} K^{p} H^{\frac{r}{2}}\right)^{w} & =\left(H^{\frac{\delta+r}{2}} T^{\frac{1}{2}}\left(T^{\frac{1}{2}} H^{\delta+r} T^{\frac{1}{2}}\right)^{\frac{1}{w}-1} T^{\frac{1}{2}} H^{\frac{\delta+r}{2}}\right)^{w} \\
& =H^{\frac{\delta+r}{2}} T H^{\frac{\delta+r}{2}} \leqslant a H^{\delta+r} .
\end{aligned}
$$

Now to show the uniqueness of $T$. Assume that $Z$ also satisfies (1.3), by (2.1) we have

$$
H^{\frac{\delta+r}{2}} Z H^{\frac{\delta+r}{2}}=\left(H^{\frac{r}{2}} K^{p} H^{\frac{r}{2}}\right)^{w}=H^{\frac{\delta+r}{2}} T H^{\frac{\delta+r}{2}},
$$

therefore $Z=T$.

It is clear that the condition (1) of Theorem 2.3 is satisfied if $H$ is invertible and the solution $T$ to (1.3) is given by $T=H^{\frac{-(\delta+r)}{2}}\left(H^{\frac{r}{2}} K^{p} H^{\frac{r}{2}}\right)^{w} H^{\frac{-(\delta+r)}{2}}$ by (2.1). In particular, if $p=r=1, \delta=0$ and $w=\frac{1}{2}$, then the unique solution to $K=T H T$ is the geometric mean $T=H^{\frac{-1}{2}}\left(H^{\frac{1}{2}} K H^{\frac{1}{2}}\right)^{\frac{1}{2}} H^{\frac{-1}{2}}$. See [13] or [2, page 11 and 105]. 


\section{Furuta's question}

In this section, as applications of Riccati equation (1.3), we prove Theorem 3.1 and Corollary 3.2 which are extensions of Theorem 1.2, and Corollary 3.3 which implies that the converse of Furuta's question is right. A generalization of a result by Lin-Cho $[15$, Theorem 2.1] is also obtained.

TheOREM 3.1. Let $C>0, D \geqslant 0$. For some fixed $r>0, \delta>-r$ and $0<w \leqslant 1$, if

$$
C^{\delta+r} \geqslant\left(C^{\frac{r}{2}} D^{s} C^{\frac{r}{2}}\right)^{w}
$$

holds for sufficiently large $s>1$. Then $D \leqslant I$.

The case $D=A^{\frac{-p}{2}} B^{p} A^{\frac{-p}{2}}$ of Theorem 3.1 ensures Corollary 3.2 immediately.

Corollary 3.2. Let $C>0, A>0, B \geqslant 0$. For each fixed $p>0, r>0, \delta>-r$ and $0<w \leqslant 1$, if

$$
C^{\delta+r} \geqslant\left(C^{\frac{r}{2}}\left(A^{\frac{-p}{2}} B^{p} A^{\frac{-p}{2}}\right)^{s} C^{\frac{r}{2}}\right)^{w}
$$

holds for sufficiently large $s>1$. Then $A^{p} \geqslant B^{p}$.

Obviously, the case $p=1, r=2$ and $\delta=-1$ of Corollary 3.2 implies that the outer exponent $\frac{1}{2}$ in Theorem 1.2 can be replaced with $w \in(0,1]$.

Proof of Theorem 3.1. By Theorem 2.3, for each sufficiently large $s>1$, there exists a unique $T(s) \geqslant 0$ satisfying $\|T(s)\| \leqslant 1$ and

$$
D^{s}=C^{\frac{\delta}{2}} T(s)^{\frac{1}{2}}\left(T(s)^{\frac{1}{2}} C^{\delta+r} T(s)^{\frac{1}{2}}\right)^{\frac{1}{w}-1} T(s)^{\frac{1}{2}} C^{\frac{\delta}{2}} .
$$

So

$$
\begin{aligned}
D^{s} & \leqslant C^{\frac{\delta}{2}} T(s)^{\frac{1}{2}}\|C\|^{(\delta+r)\left(\frac{1}{w}-1\right)} T(s)^{\frac{1}{2}} C^{\frac{\delta}{2}} \\
& \leqslant\|C\|^{\delta+(\delta+r)\left(\frac{1}{w}-1\right)} .
\end{aligned}
$$

Hence, for sufficiently large $s>1$, by Loewner-Heinz inequality ([20, page 2] or [12, page 127]) we have

$$
D \leqslant\|C\|^{\frac{w \delta+(\delta+r)(1-w)}{w s}} .
$$

By letting $s \rightarrow \infty, D \leqslant I$ follows.

It should be pointed out that the proof here is different from the proof of Theorem 1.2: the proof of Theorem 1.2 depends on Kantorovich inequality and the proof here depends on Theorem 2.3 by using Douglas's result (Lemma 2.1). 
Corollary 3.3. Let $C>0, A>0, B \geqslant 0$. For each fixed $p>0, r>p$ and $0<w \leqslant 1$, if

$$
C^{-p+r} \geqslant\left(C^{\frac{r}{2}}\left(A^{\frac{-p}{2}} B^{p} A^{\frac{-p}{2}}\right)^{s} C^{\frac{r}{2}}\right)^{w}
$$

holds for sufficiently large $s>1$. Then $A^{p} \geqslant B^{p}$.

Corollary 3.3 is the case $\delta=-p$ of Corollary 3.2 and it implies that the case $p=-t>0, r>-t$ and sufficiently large $s \geqslant 1$ of (1.4) ensures $A^{p} \geqslant B^{p}$, thus $\log A \geqslant$ $\log B$. So the converse of Furuta's question is right if (1.4) holds for $p=-t>0, r>-t$ and sufficiently large $s \geqslant 1$.

COROllary 3.4. Let $C>0, A>0, B>0$. The following assertions are equivalent to each other.

(1) $C \geqslant A \geqslant B$.

(2) If $p \geqslant 1,-1 \leqslant t<0, r>-t$ and $s \geqslant 1$, then

$$
\begin{aligned}
& C^{1+t+r} \geqslant\left(C^{\frac{r}{2}}\left(A^{\frac{t}{2}} B^{p} A^{\frac{t}{2}}\right)^{s} C^{\frac{r}{2}}\right)^{\frac{1+t+r}{(p+t) s+r}}, \\
& B^{1+t+r} \leqslant\left(B^{\frac{r}{2}}\left(A^{\frac{t}{2}} C^{p} A^{\frac{t}{2}}\right)^{s} B^{\frac{r}{2}}\right)^{\frac{1+t+r}{(p+t) s+r}} .
\end{aligned}
$$

(3) For some fixed $r>0, \delta$ and $\delta^{\prime}$ with $-r<\delta \leqslant 0$ and $-r<\delta^{\prime} \leqslant 0$, the following inequalities holds for sufficiently large $s>1$ :

$$
\begin{gathered}
C^{\delta+r} \geqslant\left(C^{\frac{r}{2}}\left(A^{\frac{-1}{2}} B A^{\frac{-1}{2}}\right)^{s} C^{\frac{r}{2}}\right)^{\frac{\delta+r}{r}}, \\
B^{\delta^{\prime}+r} \leqslant\left(B^{\frac{r}{2}}\left(A^{\frac{-1}{2}} C A^{\frac{-1}{2}}\right)^{s} B^{\frac{r}{2}}\right)^{\frac{\delta^{\prime}+r}{r}} .
\end{gathered}
$$

Corollary 3.4 is a generalization of [15, Theorem 2.1].

Proof. (1) $\Rightarrow(2)$ is the so-called grand Furuta inequality, see [9] or [19]. (3) follows by the spacial case $p=-t=1$ of (2) and Loewner-Heinz inequality for $0<$ $\frac{\delta+r}{r} \leqslant 1$ and $0<\frac{\delta^{\prime}+r}{r} \leqslant 1$. (3) ensures (1) by Corollary 3.2.

Acknowledgement. The authors would like to express cordial gratitude to the referee for his/her useful comments, especially for the improvement of Theorem 3.1.

\section{REFERENCES}

[1] E. BACH AND T. FURUTA, Counterexample to a question on the operator equation $T\left(H^{\frac{1}{n}} T\right)^{n}=K$, Linear Algebra Appl. 177 (1992), 157-162.

[2] R. Bhatia, Positive Definite Matrices, Princeton University Press, Princeton, 2007.

[3] R. Bhatia And M. UChiyama, The operator equation $\sum_{i=0}^{n} A^{n-i} X B^{i}=Y$, Expo. Math. 27 (2009) 251-255.

[4] R. G. Douglas, On majorization, factorization, and range inclusion of operators on Hilbert Space, Proc. Amer. Math. Soc. 17 (1966), 413-415. 
[5] M. FujiI, T. FURUTA And E. KameI, Furuta's inequality and its application to Ando's theorem, Linear Algebra Appl., 179 (1993), 161-169.

[6] M. FujII, E. KAMEI AND R. NAKAMOTO, On a question of Furuta on chaotic order, Linear Algebra Appl. 341 (2002), 119-127.

[7] T. FuRUtA, The operator equation $T\left(H^{\frac{1}{n}} T\right)^{n}=K$, Linear Algebra Appl. 109 (1988), 149-152.

[8] T. FURUTA, Two operator functions with monotone property, Proc. Amer. Math. Soc. 111 (1991), $511-516$.

[9] T. FURUTA, Extension of the Furuta inequality and Ando-Hiai log-majorization, Linear Algebra Appl. 219 (1995), 139-155.

[10] T. FURUTA, Results under $\log A \geqslant \log B$ can be derived from ones under $A \geqslant B \geqslant 0$ by Uchiyama's method-associated with Furuta and Kantorovich type operator inequalities, Math. Inequl. Appl., 3 (2000), 423-436.

[11] T. FURUTA, Positive semidefinite solutions of the operator equation $\sum_{j=1}^{n} A^{n-j} X A^{j-1}=B$, Linear Algebra Appl. 432 (2010) 949-955.

[12] T. FURUTA, Invitation to Linear Operators, Taylor \& Francis, London, 2001.

[13] F. Kubo And T. Ando, Means of positive linear operators, Math. Ann. 246 (1980), 205-224.

[14] P. Lancaster and L. Rodman, The algebraic Riccati equation, Oxford Academic press, 1995.

[15] C.-S. Lin And Y. J. Cho, Characterizations of the operator inequality $A>B>C$, Math. Inequal. Appl. 14 (3) (2011), 575-580.

[16] G. K. Pedersen And M. TAKes aki, The operator equation $T H T=K$, Proc. Amer. Math. Soc. 36 (1972), 311-312.

[17] G. K. Pedersen And M. TAKesaki, The Randon-Nikodym theorem for von Neumann algebras, Acta Math. 130 (1973), 53-87.

[18] J. YUAN AND Z. GAO, The operator equation $K^{p}=H^{\frac{\delta}{2}} T^{\frac{1}{2}}\left(T^{\frac{1}{2}} H^{\delta+r} T^{\frac{1}{2}}\right)^{\frac{p-\delta}{\delta+r}} T^{\frac{1}{2}} H^{\frac{\delta}{2}}$ and its applications, J. Math. Anal. Appl. 341 (2008), 870-875.

[19] J. YUAN AND G. Ji, Monotonicity of generalized Furuta type functions, Oper. Matrices 6 (2012), 809-818.

[20] X. ZHAN, Matrix Inequalities, Springer Verlag, Berlin, 2002. 